\title{
ARTICLE
}

Received 16 May 2012 | Accepted 15 Jan 2013 | Published 12 Feb 2013 DOl: 10.1038/ncomms2488

\section{Enhanced reading by training with imposed time constraint in typical and dyslexic adults}

Zvia Breznitz ${ }^{1}$, Shelley Shaul ${ }^{1}$, Tzipi Horowitz-Kraus ${ }^{1}$, Itamar Sela ${ }^{1,2}$, Michael Nevat ${ }^{1} \&$ Avi Karni ${ }^{1,2}$

Poor reading skills of developmental dyslexics persist into adulthood with standard remediation protocols having little effect. Nevertheless, reading improves if readers are induced to read faster. Here we show that this improvement can be enhanced by training. Training follows a multi-session procedure adapted to silent sentence reading, with individually set, increasingly more demanding, time constraints (letter-by-letter masking). In both typical and dyslexic adult readers, reading times are shortened and comprehension improves. After training, the dyslexic readers' performance is similar to that of typical readers; moreover, their connected text reading times and comprehension scores significantly improve in standard reading tests and are retained at 6 months post training. Identical training without time constraints proves ineffective. Our results suggest that fluent reading depends in part on rapid information processing, which then might affect perception, cognitive processing and possibly eye movements. These processes remain malleable in adulthood, even in individuals with developmental dyslexia.

\footnotetext{
${ }^{1}$ Edmond J. Safra Brain Research Center for the Study of Learning Disabilities, Department of Learning Disabilities, University of Haifa, Haifa, Israel.

${ }^{2}$ Sagol Department of Neurobiology, Department of Human Biology, University of Haifa, Haifa, Israel. Correspondence and requests for materials should be addressed to Z.B. (email: Zviab@edu.haifa.ac.il).
} 
$\mathrm{D}$ yslexic readers, including adults with university-level education, are characterized by effortful reading, which is slower and often less accurate compared to their normally reading peers ${ }^{1-3}$. The persistence of reading deficits that continues into adulthood, despite accumulating experience, has been partly attributed to a reduction in brain plasticity beyond childhood. In line with this view, it has been suggested that remedial interventions would prove less effective in adults ${ }^{1}$. Nevertheless, there is increasing evidence for effective skill learning and skill memory consolidation processes in adults ${ }^{4-9}$. It has been proposed that adults are not impaired in skill acquisition or retention per-se (including language-related skills ${ }^{8}$ ), but are rather more selective in consolidating the memory for skills compared with children or young animals ${ }^{8-12}$.

The intervention protocol that we present in the current study is based on two conceptions. First, that fluency constitutes a critical parameter of skilled reading ${ }^{13}$ and time constrained reading (that is, being forced to read at a rate faster than one's habitual reading rate) can significantly improve, albeit only for the duration of the test, reading accuracy and comprehension (the 'acceleration phenomenon') ${ }^{14,15}$. Accelerated reading may reduce distractibility, circumvent working memory limitations, increase readers' reliance on stimulus-driven word decoding ${ }^{16,17}$, enhance synchronization of brain systems ${ }^{15}$ and may reduce reliance on frontal language areas ${ }^{1,18}$. Second, training protocols, with increasingly more demanding time constraints on task performance, enhance the acquisition of a number of perceptual discrimination skills in adults $4,5,19,20,21,22$ and can improve basic processing routines $4,19,20,23$. School children, trained with speed reading, have improved reading fluency with no reduction of comprehension ${ }^{24}$.

Here we describe a computerized reading acceleration training protocol that introduced time constraints, improved the reading comprehension and reading fluency skills of both adult dyslexic readers and adult typical readers. Gains were retained 6 months post training. Importantly, the reading skills of the dyslexic group showed larger gains from the training protocol. In addition, results for testing routine reading performance (T1 test) indicate that there is a discrepancy between reading skills ability and performance for both groups. Furthermore, it is possible that the acceleration training helped to close this gap by better synchronizing between the brain systems that are activated in reading ${ }^{15}$. However, this point needs to be clarified by a study which also uses brain imaging parameters.

\section{Results}

Improved and retention of reading performance. The acceleration training $(r-a c c)$ enhanced reading performance in both standard printed reading tests and in the computerized training

Table 1 | Objective measures in standard reading tests.

\begin{tabular}{|c|c|c|c|}
\hline \multicolumn{4}{|c|}{ Connected text, comprehension (out of 30) } \\
\hline Group & $61.211, P<0.001$ & 1,109 & 0.366 \\
\hline Test time & $34.420, P<0.001$ & 2,105 & 0.396 \\
\hline Training & $0.022, P<0.968$ & 1,109 & 0.000 \\
\hline Test time $\times$ Group & $4.585, P<0.012$ & 2,105 & 0.082 \\
\hline Test time $\times$ Training & $33.370, P<0.001$ & 2,105 & 0.389 \\
\hline Group $\times$ Training & $1.134, P<0.286$ & 1,109 & 0.011 \\
\hline Group $\times$ Training $\times$ Test time & $4.472, P<0.014$ & 2,105 & 0.078 \\
\hline \multicolumn{4}{|c|}{ Connected text, word reading rate (in mis) } \\
\hline Test time & $71.391, P<0.001$ & 1,109 & 0.400 \\
\hline Training & $21.680, P<0.001$ & 2,105 & 0.177 \\
\hline Test time $\times$ Group & $21.109, P<0.001$ & 1,109 & 0.163 \\
\hline Test time $\times$ Training & $3.599, P<0.031$ & 2,105 & 0.033 \\
\hline Group $\times$ Training & $35.278, P<0.001$ & 2,105 & 0.255 \\
\hline Group $\times$ Training $\times$ Test time & $1.299, P<0.278$ & 1,109 & 0.013 \\
\hline Group $\times$ Test time $\times$ Training & 6.911, $P<004$ & 2,105 & 0.056 \\
\hline \multicolumn{4}{|c|}{ Correct words decoding per minute (in a list) } \\
\hline Group & $64.499, P<0.001$ & 1,109 & 0.510 \\
\hline Test Time & 76.767, $P<0.001$ & 2,105 & 0.594 \\
\hline Training & $20.272, P<0.001$ & 1,109 & 0.161 \\
\hline Test time $\times$ Group & $3.536, P<0.003$ & 2,105 & 0.063 \\
\hline Test time $\times$ Training & $21.885, P<0.001$ & 2,105 & 0.294 \\
\hline Group $\times$ Training & $1.617, P<0.282$ & 1,109 & 0.11 \\
\hline Group $\times$ Training $\times$ Test time & $3.158, P<0.047$ & 2,105 & 0.057 \\
\hline \multicolumn{4}{|c|}{$\begin{array}{l}\text { Statistical analyses (rm- analysis of variance) for three measures of reading (per-word reading } \\
\text { time comprehension scores in connected text reading and the number of correctly decoded } \\
\text { words in a word list) in standard reading tests (un-pointed Hebrew), using printed materials, } \\
\text { with Test time (initial, final, 6-month), as a within-Subject factor, and Group (typical, dyslexic) } \\
\text { and Training (r-acc, no-acc-read), as between-subject factors. }\end{array}$} \\
\hline
\end{tabular}

a Connected-text fluency

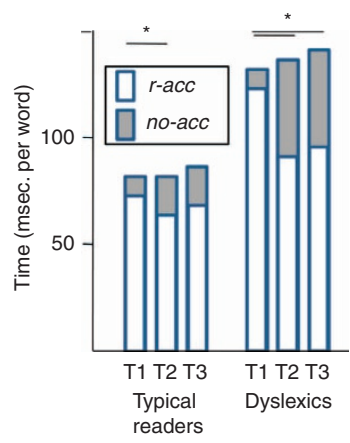

b Connected-text comprehension

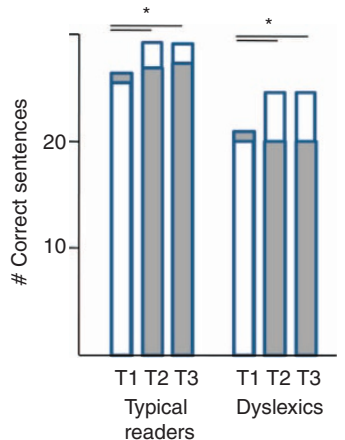

C

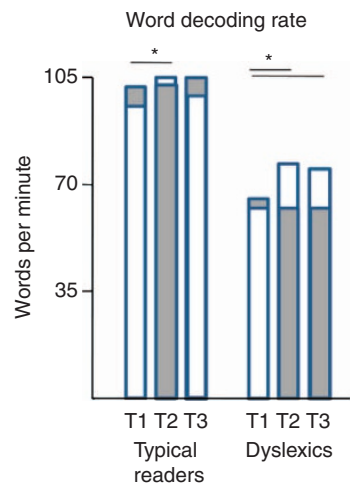

Figure 1 | Standard reading test performance of typical and dyslexic readers. Data are shown for three performance measures: (a) reading fluency for connected text; (b) number of correct responses to the comprehension questions (out of a total 30 questions); and (c) number of words decoded within a minute (word list). Note that following $r$-acc training (T2), both reading level groups, performed significantly better than the corresponding reading levelmatched individuals who underwent no-acc-read training. Moreover, the dyslexic readers who had $r$-acc training performed at a level comparable to that of the typical readers who had no $r$-acc training in terms of reading speed and comprehension of connected text. For each parameter alone, RM_analysis of variance with three test times (initial, final session and retention test at 6 months), as a within-Subject factor, and two Group (typical, dyslexic) and two Training ( $r$-acc, no-acc-read), as between-Subject, factors ${ }^{\star} P<0.05$ performed. 
protocol in two groups of readers, adult dyslexic and adult normal readers. On standard reading tests of fluency and comprehension as well as in a standard word-list reading test, readers who received $r$-acc training showed significant gains. Moreover, most of these gains were well retained in both reading level groups at 6 months post training (Table 3; Fig. 1, T3).

Although the dyslexic readers had lower scores than the typical readers, their $r$-acc training gains were significantly larger and better retained. (Table 1; Fig. 2, Figure 1 b,c). Furthermore, the performance of the dyslexics undergoing $r$-acc training was close to that of typical readers receiving no reading acceleration (no-acc-read) training on both the immediate and the delayed post-training tests (T2, T3) (Fig. 1b).

The effect of the $r$-acc training program on reading skills. The parameters of the acceleration reading training programme also indicated robust gains in reading rate and comprehension (Fig. 2a). A repeated measures analysis of variance for per-letter reading time periods with Test time (initial, final), as withinSubject, and Group (typical, dyslexic) and Training ( $r$-acc, noacc-read), as between-Subject factors, showed significant improvement across training, but also a significant reading rate difference between groups, with the dyslexics slower than the typical readers (Table 2). The type of training afforded was critical, as the gains in reading rate were exclusively expressed in participants training with time-constrained reading $(r$-acc) (Table 2) (Fig. 2a). Overall, $r$-acc training was equally effective in the two reading level groups, but there were no significant gains in the no-acc-read training protocol (Fig. 2a).

During training, the comprehension scores in reading-masked sentences also improved significantly in all participants receiving $r$-acc training $(76 \pm 6 \%$ to $87 \pm 7 \%, 89 \pm 4 \%$ to $97 \pm 5 \%$, average \pm s.d., percent correct responses to comprehension questions within the initial and final sessions, dyslexic and typical readers, respectively), (Table 2) (Fig. 2b). No such gains were found in either reading level group after no-acc-read training (76 $\pm 7 \%$ to $75 \pm 8 \%, 91 \pm 4 \%$ to $89 \pm 3 \%$, percent correct responses in initial and final sessions, dyslexic and typical readers, respectively), so that the gains accrued only in $r$-acc training (Table 2). The typical readers had significantly higher comprehension scores both before and after training (Table 2; Fig. 2b). However, the dyslexics gained more from $r$-acc training, compared with the typical readers.

The training test parameters indicated that before training, the dyslexic readers assigned to $r$-acc training were significantly slower $(t(1,79)=5.97, P<0.001)$ and scored lower on the comprehension questions $(t(1,79)=4.21, P<0.001)$ compared with typical readers, with the slower reading rates negatively correlated with comprehension $(R=-0.598, P<0.001)$. However, in the
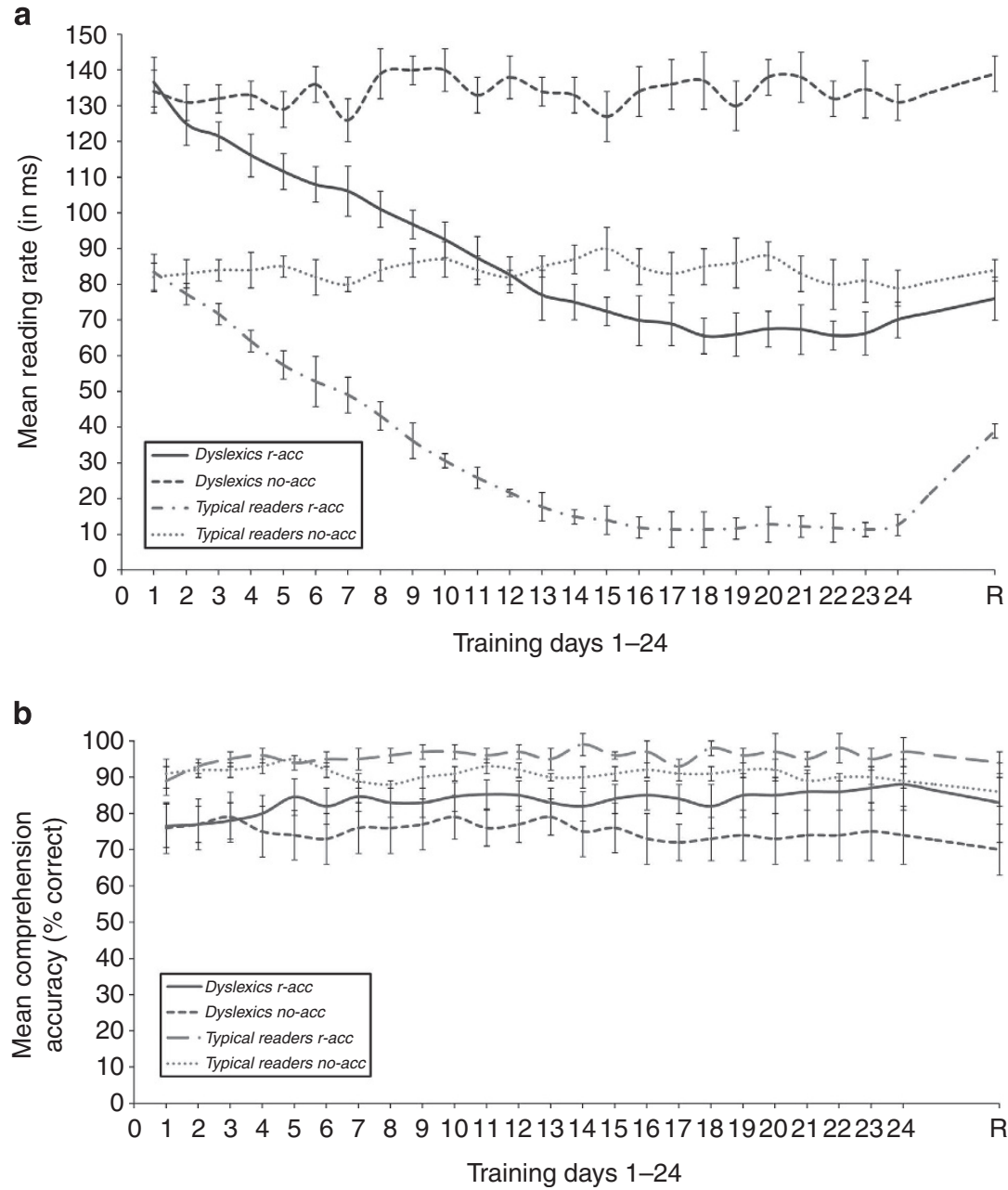

Figure 2 | The effects of $\boldsymbol{r}$-acc practice across 24 training sessions in typical and dyslexic readers. Group means for (a) reading rates and (b) comprehension accuracy. The per-letter reading rate was computed as the sentence reading time divided by the number of characters in the sentence. Comprehension accuracy is shown as a percentage of correct responses to the comprehension questions. Error bars represent standard deviation of the group means. 
Table 2 | Training and long-retention interval effects.

\section{Task measure}

\section{Main effects and interactions}

$\mathbf{F}, \mathbf{P}$

df

169.242, $P<0.001$

15.779, $P<0.001$

$28.855, P<0.001$

2.399, $P<0.129$

40.487, $P<0.001$

14.700, $P<0.001$

$0.344, P<0.561$

40.383, $P<0.001$

56.444, $p<0.001$

18.361, $P<0.001$

1.733, $P<0.191$

$72.908, P<0.001$

$0.291, P<0.590$

$0.024, P<0.877$
1,109

1,109

1,109

1,109

1,109

1,109

1,109

1,109

1,109

1,109

1,109

1,109

1,109

1,109
Partial $\eta$ Squared

0.615

0.130

0.214

0.022

0.276

0.122

0.003

0.276

0.346

0.148

0.016

0.408

0.003

0.000

(b) Retention of training effects: final session versus retention test at 6 months post training

Reading comprehension

Group
Test-time
Training
Test-time $\times$ Group
Test time $\times$ Training
Group $\times$ Training
Group $\times$ Test-time $\times$ Training
Group
Test-time
Training
Test-time $\times$ Group
Test Time $\times$ Training
Group $\times$ Training
Group $\times$ Test-time $\times$ Training

145.416, $P<0.001$

51.345, $P<0.001$

78.319, $P<0.001$

123.323, $P<0.001$

78.044, $P<0.001$

17.262, $P<0.001$

15.203, $P<0.006$

48.264, $P<0.001$

37.080, $P<0.001$

46.634, $P<0.001$

1.414, $P<0.273$

28.900, $P<0.001$

1.376, $P<0.243$

$0.139, P<0.710$

$\begin{array}{ll}1,109 & 0.579 \\ 1,109 & 0.541 \\ 1,109 & 0.525 \\ 1,109 & 0.421 \\ 1,109 & 0.440 \\ 1,109 & 0.140 \\ 1,109 & 0.125 \\ 1,109 & 0.319 \\ 1,109 & 0.259 \\ 1,109 & 0.305 \\ 1,109 & 0.013 \\ 1,109 & 0.214 \\ 1,109 & 0.013 \\ 1,109 & 0.001\end{array}$

(a) Statistical analyses ( $r m$ - analysis of variance s) for (i) comprehension accuracy (\% correct responses) and (ii) reading speed (per-letter reading time), with Test time (initial, final session), as a within(a) Statistical analyses ( $r m$ - analysis of variance s) for ( $i)$ comprehension accuracy (\% correct responses) and
Subject factor, and Group (typical, dyslexic) and Training ( $r$-acc, no-acc-read), as between-Subject factors.

(b) An identical analysis with Test time (final session, retention test at 6 months post training), as the within-Subject factor.

\section{Table 3 | Reading and general ability measures at enrolment.}

\begin{tabular}{|c|c|c|c|c|}
\hline & $\begin{array}{c}\text { Dyslexics } N=55 \\
\text { Mean (s.d.) }\end{array}$ & $\begin{array}{c}\text { Typical readers } N=55 \\
\text { Mean (s.d.) }\end{array}$ & $\mathbf{F}$ & $P$-value \\
\hline Decoding (Z scores) & $-1.26(0.34)$ & $0.85(0.51)$ & 57.39 & 0.001 \\
\hline Comprehension ( $Z$ scores) & $-0.25(1.06)$ & $0.51(0.19)$ & 7.66 & 0.002 \\
\hline Phonological processing time ( $Z$ scores) & $-0.72(0.45)$ & $0.65(0.33)$ & 45.21 & 0.001 \\
\hline Phonological processing accuracy ( $Z$ scores) & $-0.19(1.05)$ & $0.21(0.87)$ & 5.04 & 0.027 \\
\hline Rapid letter naming time ( $Z$ scores) & $-0.66(1.04)$ & $0.59(0.27)$ & 76.47 & 0.001 \\
\hline Working memory (words) & $-54(0.65)$ & $0.59(0.98)$ & 51.43 & 0.001 \\
\hline Raven (standard score) & $61.46(17.51)$ & $59.43(18.33)$ & 0.39 & 0.54 \\
\hline Block design (standard score) & $12.21(2.27)$ & $12.79(2.77)$ & 1.54 & 0.22 \\
\hline Similarities (standard score) & $8.77(1.30)$ & $8.91(1.20)$ & 0.35 & 0.56 \\
\hline DSM-ADHD ( $Z$ score) & $0.48(0.95)$ & $0.50(0.98)$ & 1.22 & 0.29 \\
\hline
\end{tabular}

final training sessions, dyslexic participants who had $r$-acc training had similar scores to the typical readers who had no-accread training, both in their per-letter reading rates and in the accuracy of responses to comprehension questions (Fig. 2). Moreover, the gains were retained over a 6-month interval with the dyslexics showing somewhat better retention in terms of comprehension (Table 2b; Fig. 2).

\section{Discussion}

The imposition of time constraints on text reading during training was a crucial factor in improving reading skill in both reading-level groups. There are grounds to consider the possibility that the beneficial effects of training with time constrained masking relate to modifications of cognitive, motor, perceptual or word decoding routines specific to the skill of 
reading ${ }^{25-27}$. Poor reading is characterized by atypical ocular motor routines in reading ${ }^{28}$, such as more regressions and longer fixations, ${ }^{29,30}$ and these routines may also be modified by training $29-33$. In adults, time-constrained training protocols have proved to be highly effective in inducing long-lasting gains in visual processing speed while improving discrimination ${ }^{4,5,19,21}$. Ineffective reading routines are implicated in explaining the discrepancy between accelerated and standard reading performance in dyslexic readers ${ }^{15}$. However, as our consistently negative results in the no-acc-read condition suggest, training in reading from a computer screen without imposed time constraints may engage the previously well-established reading routines (motor, perceptual or both), irrespective of reading level, in adults, rather than induce the establishment of new sub-routines. Nevertheless, training under task conditions that are far removed from the standard real-life reading experience may lead to expertise, which cannot be applied to standard reading performance $e^{4,22,34-36}$. Thus, the significant transfer of the $r$-acc-related gains to everyday text-reading performance indicates that the training experience engaged a level of processing that is of relevance to normal, unmasked and connected-text reading. In addition, the improvement of single word decoding as a result of the $r$-acc training among the two reading level groups may indicate a better access to and retrieval of word patterns from the mental lexicon and thus, may enhance connected-text reading rate and comprehension.

Our findings also indicated that long-lasting retention of faster reading rate and higher comprehension was found within the two groups after $r$-acc training. The fact that the retention of the training effect was higher among the dyslexic readers, could instead of can be due to their lower initial reading skills, allowing them to gain much more in reading rate and comprehension.

The current results indicate that the experience of reading with demanding, but manageable, time constraints may facilitate the establishment of additional improved text-processing subroutines, even in highly experienced adults. Furthermore, our results indicate a behaviourally relevant potential for improving reading skills in adult dyslexics. Nevertheless, as the gap between the dyslexics' performance vis-à-vis trained typical readers was clearly maintained after training, the current results suggest that $r$-acc, although highly effective, may not reset the life-long reading disability, but rather that a less-than-optimal reading routine was made more effective, for the dyslexics. This study focused adult, compensated dyslexics, and whether the training would bring about similar results for children must still be verified.

\section{Methods \\ Subjects. A total of 110 university students, 55 recognized as dyslexic by the University of Haifa Clinic (Israeli Ministry of Education criteria, 2004), partici- pated in the study. All dyslexic participants achieved mean reading scores of -1 s.d. and less in MATAL (2007) ${ }^{39}$ standardized reading achievement test in Hebrew. The participants of both reading level groups (typical, dyslexic) were matched for age (22-29 years), gender (1:2, males/females), non-verbal IQ and level of education (2-4 years at the University) and had no indication of an attention deficit disorder (DSM 1994) ${ }^{37}$. Reading and reading-related standard tests performed before inclusion in the study showed that the dyslexics were significantly underperforming in all reading-related tests as well as in working memory capacity for words (Table 3 ).}

Training protocol. Forty participants of each reading level group were assigned to training in sentence reading with increasingly demanding time constraints (reading acceleration, $r-a c c)$; 15 participants in each reading level group were assigned an identical amount of reading practice, with identical sentences, but with no time constraints (self-paced reading, no-acc-read). All participants received 24 practice sessions (15-20 min each), three sessions per week with 1-3 days between-session intervals. In each session, 50 sentences (9-12 words, 45-70 letters, per sentence) were read, silently. The words were of medium-to-low frequency, (http:// atar.mscc.huji.ac.il/ frost/files/Word_frequency.xls) in the un-pointed Hebrew script format. Each sentence was presented once throughout training. Participants were instructed to press a key as soon as they finished reading each sentence. A multiple-choice comprehension question followed.

In order to overcome possible word length effects, time constraints (masking, $r$-acc) were imposed using letter-by-letter erasure, advancing in the reading direction. Initial per-letter erasure rates were determined individually, from per-letter reading times in a 12 sentences pre-test (self-paced silent reading). Thereafter, erasure rate was increased or decreased by $2 \mathrm{~ms}$ per-character according to a stair-case procedure based on the number of consecutive comprehension questions correctly answered. Pre, post and long-post standard reading tests were obtained. Word decoding accuracy (Shatil, 1995) ${ }^{38}$, reading time for connected text and reading comprehension (MATAL, 2007) ${ }^{39}$ were measured for all participants.

\section{References}

1. Shaywitz, S. E. \& Shaywitz, B. A. Paying attention to reading: the neurobiology of reading and dyslexia. Dev. Psychopathol. 20, 1329-1349 (2008).

2. Vellutino, F. R., Fletcher, J. M., Snowling, M. J. \& Scanlon, D. M. Specific reading disability (dyslexia): What have we learned in the past four decades? J. Child Psychol. Psychiatry 45, 2-40 (2004).

3. Lefly, D. L. \& Pennington, B. F. Spelling errors and reading fluency in compensated adult dyslexics. Ann. Dyslexia 41, 143-161 (1991).

4. Karni, A. \& Sagi, D. Where practice makes perfect in texture discrimination: evidence for primary visual cortex plasticity. Proc. Natl Acad. Sci. USA 88, 4966-4970 (1991).

5. Karni, A. \& Sagi, D. The time course of learning a visual skill. Nature 365, 250-252 (1993).

6. Buonomano, D. V. \& Merzenich, M. M. Cortical plasticity: From syanpses to maps. Annu. Rev. Neurosci. 21, 149-186 (1998).

7. Karni, A., Meyer, G., Rey-Hipolito, C., Jezzard, P., Adams, M. M., Turner, R. \& Ungerleider, L. G. The acquisition of skilled motor performance: fast and slow experience-driven changes in primary motor cortex. Proc. Natl Acad. Sci. USA 95, 861-868 (1998).

8. Ferman, S. \& Karni, A. No childhood advantage of skill in using an artificial language rule. PLoS ONE 5, e13648v (2010).

9. Weinberger, N. M. \& Bakin, J. S. Learning-induced physiological memory in adult primary auditory cortex: receptive fields plasticity, model, and mechanisms. Audiol. Neurootol. 3, 145-167 (1998).

10. Dorfberger, S., Adi-Japha, E. \& Karni, A. Reduced susceptibility to interference in the consolidation of motor memory before adolescence. PLOS ONE 2, 1-6 (2007).

11. Keuroghlian, A. S. \& Knudsen, E. I. Adaptive auditory plasticity in developing and adult animals. Prog. Neurobiol. 82, 109-121 (2007).

12. Edeline, J. M. Learning-induced physiological plasticity in the thalamo-cortical sensory systems: a critical evaluation of receptive field plasticity, map changes and their potential mechanisms. Prog. Neurobiol. 57, 165-224 (1999).

13. National Institute of Child Health and Human Development (NICHD). Report of the National Reading Panel. Teaching children to read: an evidence-based assessment of the scientific research literature on reading and its implications for reading instruction: Reports of the subgroups (NIH Publication No. 00-4754). US Government Printing.

14. Breznitz, Z. Increasing first graders' reading accuracy and comprehension by accelerating their reading rates. J. Educ. Psychol. 79, 236-242 (1987).

15. Breznitz, Z. Reading Fluency: Synchronization of Processes. Lawrence Erlbaum and Associates (2006).

16. Breznitz, Z. The effect of accelerated reading rate on memory for text among dyslexic readers. J. Educ. Psychol. 89, 287-299 (1997).

17. Breznitz, Z. \& Share, D.L. The effect of accelerated reading rate on memory for text. J. Educ. Psychol. 84, 193-200 (1992)

18. Karni, A., Morocz, I.A., Bitan, T., Shaul, S., Kushnir, T. \& Breznitz, Z. An fMRI study of the differential effects of word presentation rates (reading acceleration) on dyslexic readers' brain activity patterns. J. Neurolinguistics 18, 197-219 (2005).

19. Fahle, M. \& Edelman, S. Long-term learning in vernier acuity: effects of stimulus orientation, range and of feedback. Vis. Res. 33, 397-412 (1993).

20. Levi, D., Polat, U \& Hu, YS Improvement in Vernier acuity in adults with amblyopia. Practice makes better. Investigative ophthalmology. Vis. Sci. 38, 1493-1510 (1997).

21. Farmer, M. \& Klein, R. M. The evidence for a temporal processing deficit linked to dyslexia: A review. Psychon. Bull. Rev. 2, 460-493 (1995).

22. Eden, G. \& Moats, L. The role of Neuroscience in the remediation of students with dyslexia. Nat. Neurosci. Suppl 5, 1080-1084 (2002).

23. Schwartz, S, Maquet, P \& Frith, C. Neural correlates of perceptual learning: functional MRI study of visual texture discrimination. Proc. Natl Acad. Sci. USA. 2002 99, 17137-17142 (2002).

24. Irausquin, R. S., Drent, J. \& Verhoeven, L. Benefits of Computer-presented speed training for poor readers. Ann. Dyslexia 55, 246-265 (2005).

25. Tallal, P. Improving language and literacy is a matter of time. Nature Rev. Neurosci. 5, 721-728 (2004). 
26. Stein, J. \& Walsh, V. To see but not to read; the magnocellular theory of dyslexia. Trends Neurosci. 20, 147-152 (1997).

27. Tallal, P. The science of literacy: from the laboratory to the classroom. Proc. Natl Acad. Sci. USA 97, 2402-2404 (2000)

28. Stein, J. Evaluation of an exercise based treatment for children with reading difficulties. Dyslexia 9, 48-71 (2003).

29. Tran, K., Yu, C., Okumura, T. \& Laukkanen, H. Effectiveness of an on-line computerized eye movement training program to improve oculomotor control in adult readers: a pilot study. J. Behav. Optom. 15, 115-121 (2004).

30. Radach, R., Vorstius, C. \& Reilly, R. The science of speed reading: exploring the impact of speed on visuomotor control and comprehension (Paper presented at the Annual Meeting of the Society for the Scientific Study of Reading, Berlin, Germany, 10 July 2010, 2010)

31. Judica, A, De Luca, M., Spinelli, D. \& Zoccolotti, P. Training of developmental surface dyslexia improves reading performance and shortens eye fixation duration in reading. Neuropsychol. Rehabil. 12, 177-197 (2002).

32. De Luca, M., Borrelli, M., Judica, A., Spinelli, D. \& Aoccolotti, P. Reading words and pseudowords: an eye movement study of developmental dyslexia. Brain Lang. 80, 617-626 (2002).

33. Leff, A.P. \& Behrmann, M. Treatment of Reading impairment after stroke. Curr. Opin. Neurol. 21, 644-648 (2008)

34. Talcott, J. B. et al. Dynamic sensory sensitivity and children's word decoding skills. Proc. Natl Acad. Sci. USA 97, 2952-2957 (2000).

35. Poggio, T. \& Bizzi, E. Generalization in vision and motor control. Nature 431, 768-774 (2004)

36. Owen, A. M. et al. Putting brain training to the test. Nature 465, 775-778 (2010)

37. American Psychiatric Association. Diagnostic and Statistical Manual of Mental Disorders: DSM-1V (American Psychiatric Press, 1994).
38. Shatil, E. One-Minute Test for Words (University of Haifa, Haifa, 1995). 39. MATAL. Diagnostic battery for the assessment of learning abilities (Center for Psychometric Tests, Jerusalem, 2007)

\section{Acknowledgements}

This research was supported by a grant from the Zeit Foundation and the Edmond J. Safra Philanthropic Foundation. The study was approved by the Institutional Review Board, University of Haifa (090/09) and by the Ethics Committee, C. Sheba Medical Center.

\section{Author contribution}

Z.B. was the creator of the research idea, she was the head of the research group carrying out the study and summarized the research. A.K. was a co-investigator for this study and contributed to summarizing the project. The remaining authors were research assistants who helped with data collection. M.N. was the computer programmer for the $r$-acc programme.

\section{Additional information}

Competing financial interests: The authors declare no competing financial interests.

Reprints and permission information is available at http://npg.nature.com/ reprintsandpermissions.

How to cite this article: Breznitz, Z. et al. Enhanced reading by training with imposed time constraint in typical and dyslexic adults. Nat. Commun. 4:1486 doi: 10.1038/ ncomms2488 (2013). 